Article

\title{
Numerical Simulation and Mechanism Analysis on the Concave Deformation of Automotive Dry Clutch Pressure Plate
}

\author{
Yubing Gong ${ }^{1, *}$, Penghui Wang ${ }^{1}$, Wencheng Ge ${ }^{1}$ and Yun-Bo $\mathrm{Yi}^{2}$ \\ 1 School of Mechanical \& Electrical Engineering, Guilin University of Electronic Technology, Guilin 541004, \\ China; wphmk6@163.com (P.W.); gewc123@163.com (W.G.) \\ 2 Department of Mechanical and Materials Engineering, University of Denver, Denver, CO 80208, USA; \\ Yun-Bo.Yi@du.edu \\ * Correspondence: gybcome@guet.edu.cn
}

Received: 10 September 2019; Accepted: 16 November 2019; Published: 21 November 2019

\begin{abstract}
It is well known that slipping in automotive clutches is closely related to the concave deformation of the pressure plate, but the mechanism of this deformation is not clear. A simplified model of a pressure plate assembly is developed by simulating the deformation field of the plate under the condition of continuous sliding, followed by natural cooling to room temperature. The distribution of displacement is obtained from numerical simulations. The equivalent von Mises stresses of the pressure plate with or without concave deformation are analyzed and compared. The mechanism leading to the concave deformation of the pressure plate is put forward in terms of equivalent von Mises stress. The results show that (1) the concave deformation of the pressure plate is produced during the cooling process and (2) the profile of the concave deformation is determined by its thermal stress distribution. The deformation is mainly caused by the overstressed upper part of the inner rim of the pressure plate during clutch operations.
\end{abstract}

Keywords: pressure plate; concave deformation; thermal stress; finite element

\section{Introduction}

Dry clutch is one of the important parts of automotive transmission systems. Meanwhile, the pressure plate is one of the main components of a dry clutch. When a clutch is in operation, the torque from the engines is transmitted by the sliding of the pressure plate against the friction plate. The frictional heat introduced by the relative motion increases the temperature of the pressure plate, which normally increases the thermal stress and deformation of pressure plate, sometimes resulting in the failure of the clutch [1,2]. As the deformation of the pressure plate, especially the concave deformation of the pressure plate used for heavy duty, has an obvious relationship with the clutch slipping, it is often required that the maximum concave deformation of the pressure plate be below some specific threshold value in the clutch applications.

Yantian Sheng-san [3] obtained the tendency relationship between the deformation of the pressure plate and the temperature during the slip period of the clutch engagement, and observed that the friction surface of pressure plate appears in "drum" type (internal convex) when heating and in "dish" type (internal concave) when cooling. Hang and Zhu [4] et al. used the finite element method to simulate the drum type thermal deformation under the starting condition of the pressure plate. Hang [5] et al. simulated the temperature field and the axial (drum) deformation of the friction surface of a heated pressure plate. Through the finite element method, Zhu [6] and others also obtained the "drum-shaped" thermal deformation of the pressure plate in the clutch engagement process. 
A two-dimensional axisymmetric model was established by Abdullah et al., and the distribution of temperature field during the 10-times process of clutch pressure plate engagement was analyzed under the two assumptions of uniform pressure distribution and linear pressure distribution [7]; the temperature field distributions of the pressure plate under three different contact areas- $100 \%, 75 \%$, and $50 \%$-between the pressure plate and the friction plate were also analyzed and compared [8]. Abdullah et al. [9] used the thermomechanical coupling method to analyze the radial temperature distribution of the friction surface of the pressure plate under different slipping speeds. Also, Abdullah et al. [10] performed the thermal analysis on the flywheel clutch disc, and pointed out that the deformation of pressure plate approximately increases along the radial direction. Wang and Zhang [11,12] conducted numerical simulation of temperature field and thermal stress field under different loading conditions. Gong and others [13] discussed the effect of thermal parameters and structural parameters on the temperature field and deformation field of pressure plate using the response surface method, and proposed a new structure of the pressure plate. Gong and others [14] provided evidence that the concave deformation of pressure plate is not induced by the thermal buckling mechanism through thermal buckling simulation.

It can be seen that there are few researches on the concave deformation of the pressure plate, and its mechanism is not clear yet. In this paper, a simplified model of the assembly of the pressure plate is developed, and the evolution of the deformation of the pressure plate during frictional heating and cooling is simulated by thermal mechanical coupling. The distributions of stress in the radial and axial directions of the pressure plate during these processes are obtained and the internal concave deformation is analyzed. The equivalent von Mises stress of pressure plate is compared with the results without internal concave deformation. The characteristics of the stress distribution of the pressure plate are obtained, and the critical distribution of stress causing the concave deformation of the press plate is discussed.

\section{Finite Element Model of Pressure Plate Assembly}

\subsection{Finite Element (FE) Model}

In the existing literature on the finite element modeling of pressure plates, the fixtures on the edges of the pressure plate assembly were rarely considered due to the extra computational effort involved. However, this approach (without considering the fixtures) failed to capture the details in the realistic boundary condition, $r$. To improve the accuracy, an improved computational model of the pressure plate assembly shown in the following Figure 1 established. Particularly, the assembly model contains the pressure plate, diaphragm springs, rivets, as well as the transmission plates.

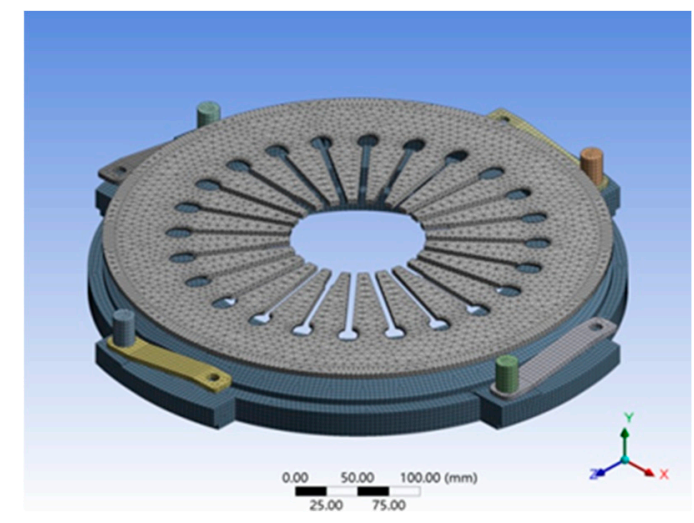

Figure 1. Simplified model of pressure plate assembly.

In this assembly FE model, according to the practical physical interaction between the pressure plate and other parts, the nonlinear boundary conditions of the pressure plate are incorporated. 
The contact between the pressure plate and the diaphragm spring is treated as a frictional contact, whereas the contact between the pressure plate and the drive plate is simulated as a bonded contact. The contacts between the pressure plate and the rivet areas also simulated as bonded contacts. The entire geometry is meshed with elements of $4 \mathrm{~mm}$ size. There are 445,027 nodes and 127,561 elements totally.

\subsection{Boundary Conditions}

\subsubsection{Thermal Boundary Conditions}

In the continuous sliding condition, it is assumed that the relative speed between the clutch pressure plate and the driven plate is invariable and the pressure plate always receives more heat than the friction plate. The power of the shaded area (as shown in Figure 2) is calculated as follows when the pressure plate is under the sliding condition [15],

$$
\mathrm{dp}=\frac{F(t)}{\pi\left(r_{2}^{2}-r_{1}^{2}\right)} \cdot 2 \pi r d r \cdot \mathrm{f} r \omega_{h}
$$

where $\mathrm{p}$ is the sliding power of the shaded area; $r_{1}$ and $r_{2}$ inner radius and outer radius of pressure plate, respectively; $r$ is the radius where the shaded area is located in the friction surface; $F(t)$ is the clamping load of pressure plate; $\mathrm{f}$ is the coefficients of friction; $\omega_{h}$ is the angular velocity difference between the pressure plate and the friction surface; and $2 \pi r d r$ is the area of the shaded portion.

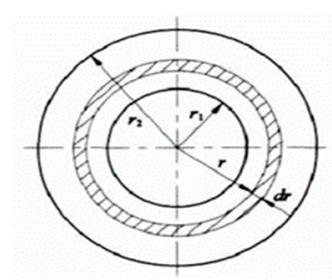

Figure 2. Friction surface of the pressure plate.

In the continuous sliding condition, as the clamping load of pressure plate and the relative angular velocity between the pressure plate and the driven disk remain unchanged, we have

$$
\begin{gathered}
\mathrm{F}(\mathrm{t})=\mathrm{N} \\
\omega_{h}=\omega
\end{gathered}
$$

where $\mathrm{N}$ and $\omega$ are constant.

Then, Equation (1) can be rewritten as Equation (4) after substituting Equations (2) and (3) into Equation (1):

$$
\mathrm{dp}=\frac{\mathrm{fN} \omega \mathrm{r}}{\pi\left(r_{2}^{2}-r_{1}^{2}\right)} \cdot 2 \pi r d r
$$

where $\frac{\mathrm{fN}}{\pi\left(r_{2}^{2}-r_{1}^{2}\right)} \cdot 2 \pi r d r$ represents the friction forces of the shaded area, and $\omega \mathrm{r}$ is the linear velocity of the shaded area.

By definition [16], the heat flux on the friction surface of pressure plate can be obtained as Equation (5):

$$
\mathrm{q}=\frac{\mathrm{dp}}{\mathrm{ds}}=\frac{\frac{\mathrm{fN} \omega \mathrm{r}}{\pi\left(\mathrm{r}_{2}^{2}-\mathrm{r}_{1}^{2}\right)} \cdot 2 \pi \mathrm{rdr}}{2 \pi \mathrm{rdr}}=\frac{\mathrm{fN} \omega \mathrm{r}}{\pi\left(\mathrm{r}_{2}^{2}-\mathrm{r}_{1}^{2}\right)}
$$


The total heat flux induced by the relative sliding is absorbed by the pressure plat and the driven plate. The heat distribution coefficient between them follows Equation (6),

$$
\mathrm{k}=\frac{\mathrm{q}_{\text {Pressureplate }}}{\mathrm{q}_{\text {frictionplate }}}=\left(\frac{\lambda_{\text {Pressureplate }} \mathcal{C}_{\text {Pressureplate }} \rho_{\text {Pressureplate }}}{\lambda_{\text {frictionplate }} \mathcal{C}_{\text {frictionplate }} \rho_{\text {frictionplate }}}\right)^{0.5}
$$

where $\lambda$ is the thermal conductivity, $c$ is the thermal capacity, and $\rho$ is the density.

Therefore, the heat flux on the friction surface of the pressure plate after the heat distribution coefficient is taken into account can be expressed as

$$
\mathrm{q}_{\text {Pressure plate }}=\frac{\mathrm{qk}}{1+\mathrm{k}}
$$

In our case, here the parameters needed have been provided by the clutch company, and the heat flux of friction surface of pressure plate is $q_{\text {Pressure plate }}=2,188,000 \mathrm{r} \mathrm{J} /\left(\mathrm{m}^{2} \cdot \mathrm{s}\right)$.

According to the condition of actual continuous sliding, the slip time of pressure plate is set to $60 \mathrm{~s}$, and a natural convection cooling process of $3500 \mathrm{~s}$ is subsequently applied at room temperature $\left(25^{\circ} \mathrm{C}\right)$. During the cooling process, the convective heat transfer coefficient on the surface of the pressure plate is estimated as [17]

$$
\mathrm{h}=0.023 \frac{\lambda}{d} R_{e}^{0.8} p_{r}^{0.4}
$$

where $\lambda$ is the air thermal conductivity, $d$ is the characteristic size, $R_{e}$ is the Reynolds number, and $p_{r}$ is the Prandtl number.

\subsubsection{Constraint Conditions}

To analyze the concave deformation characteristics of the pressure plate, two different types of constraints are considered here: The first condition is shown in Figure 3, and the other one is shown in Figure 4 below.

In both constraint conditions, the contact area of the diaphragm spring that touches the support ring is a fixed constraint, labeled " $\mathrm{A}$ " in Figures 3 and 4 . The bolt hole in drive plate which connects the drive plate and the clutch cover is also fixed in all degrees of freedom (i.e., DOEs), shown as label " $\mathrm{B}$ " in Figure 3. The clamping force here, 36,000 N, is applied on the friction surface of the pressure plate labeled " $C$ " in Figures 3 and 4.

In the second type of constraint condition, the upper surface of the transmission plate and the rivet is fixed, which is labeled " $\mathrm{D}$ " in Figure 4. This constraint is the only different constrain between the first type boundary conditions (BCs) and the second type BCs. The other three constraints are the same.
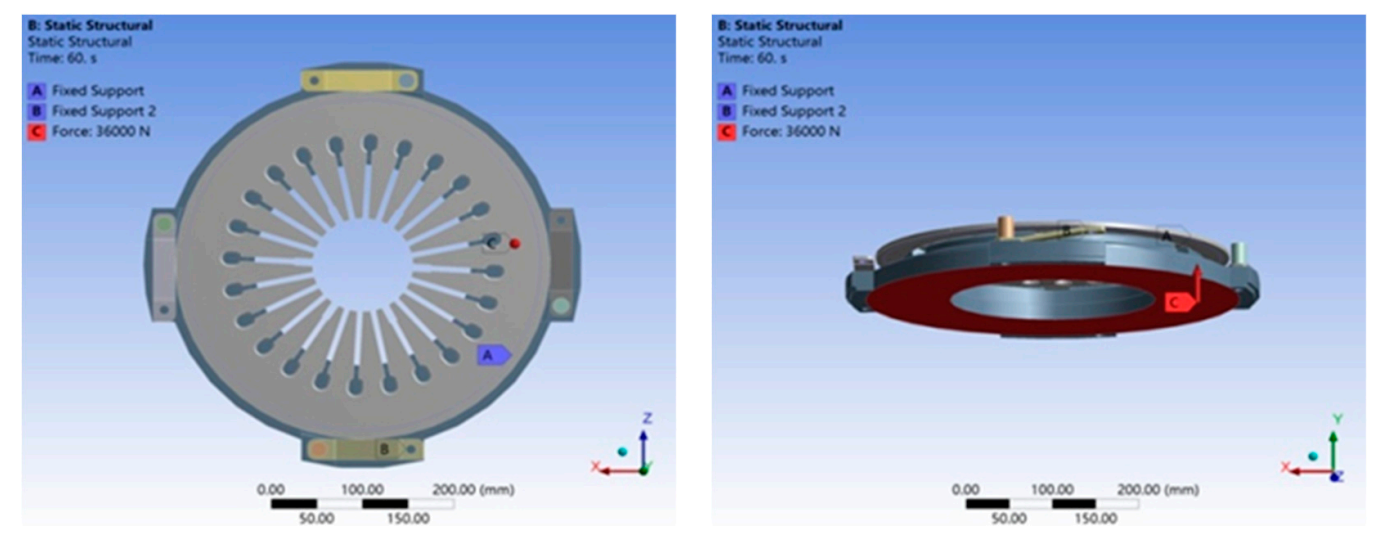

Figure 3. Constraint 1 . 

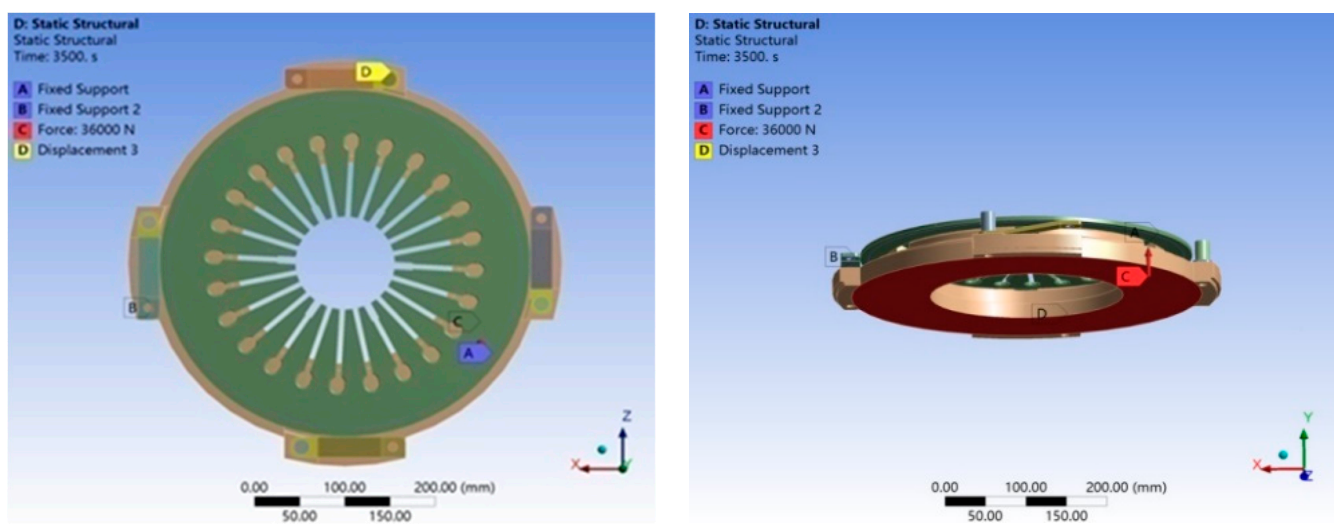

Figure 4. Constraint 2 .

\subsection{Surface Thermal Stress of General Ring}

For a ring, given a specific temperature distribution, the resulting circumferential and radial thermal stresses of the ring can be computed by the following Formulas (9) and (10), respectively,

$$
\begin{gathered}
\sigma_{\theta}=\frac{\alpha \cdot E}{r^{2}} \cdot\left\{\frac{r^{2}+r_{1}^{2}}{r_{2}^{2}-r_{1}^{2}} \cdot \int_{r_{1}}^{r_{2}} T(r) \cdot r \cdot d r+\int_{r_{1}}^{r} T(r) \cdot r \cdot d r+[T(r)]^{2}\right\} \\
\sigma_{r}=\frac{\alpha \cdot E}{r^{2}} \cdot\left\{\frac{r^{2}-r_{1}^{2}}{r_{2}^{2}-r_{1}^{2}} \cdot \int_{r_{1}}^{r_{2}} T(r) \cdot r \cdot d r+\int_{r_{1}}^{r} T(r) \cdot r \cdot d r\right\}
\end{gathered}
$$

where $\alpha$ is the thermal expansion coefficient of the ring, $E$ is the Yong's modulus, $r_{1}$ is the inner radium, and $r_{2}$ is the outer radium. For the complexity of the temperature distribution and constrain conditions of the pressure plate during the engagement stage, the thermal stress in pressure plate is hardly solved by the above formula and the FE simulation is used to obtain the thermal stress.

\section{Results of Finite Element Analysis of Pressure Plates}

\subsection{Analysis of Deformation of Pressure Plate}

The temperature distribution and the consequently thermal deformation distribution of the pressure plate in the processes of heating and cooling are obtained based on the finite element model. At several critical moments, such as $60 \mathrm{~s}, 695 \mathrm{~s}$, and $3500 \mathrm{~s}$, the temperature distribution and thermal deformation distribution of the pressure plate are shown in Figures 5-7, respectively.

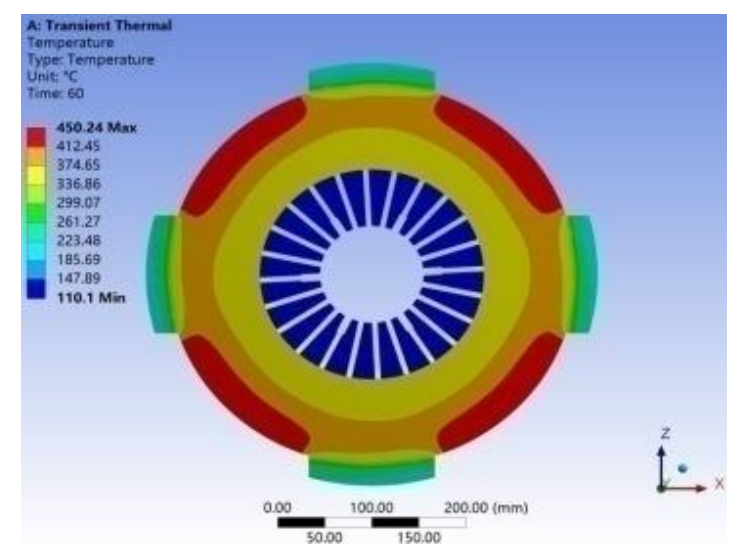

(a)

Figure 5. Cont. 


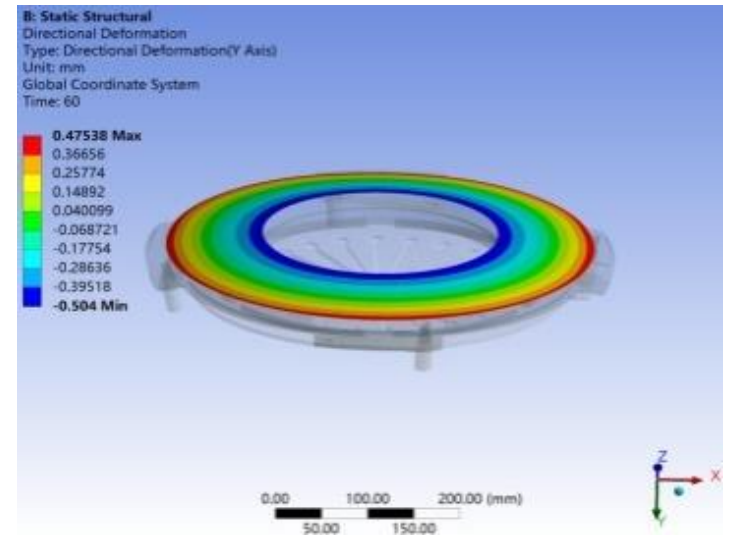

(b)

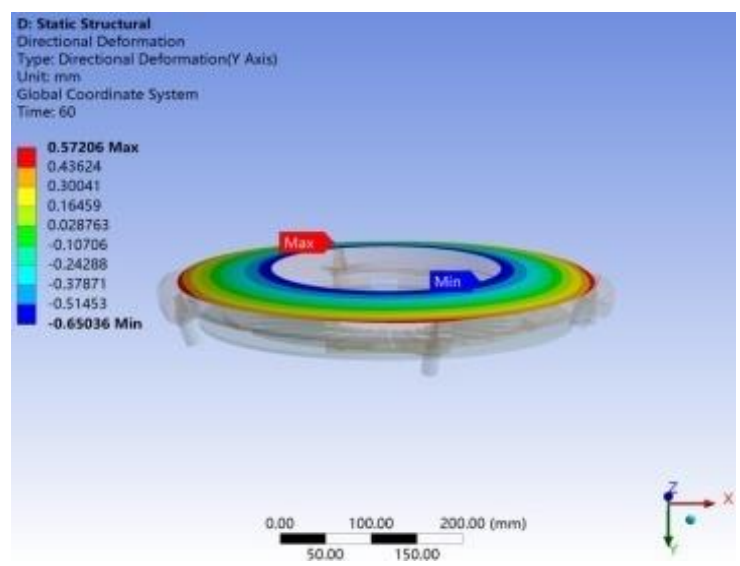

(c)

Figure 5. The temperature field (a), deformation field under constraint condition 1 (b), and deformation field under constraint condition 2 (c) of pressure plate at $60 \mathrm{~s}$.

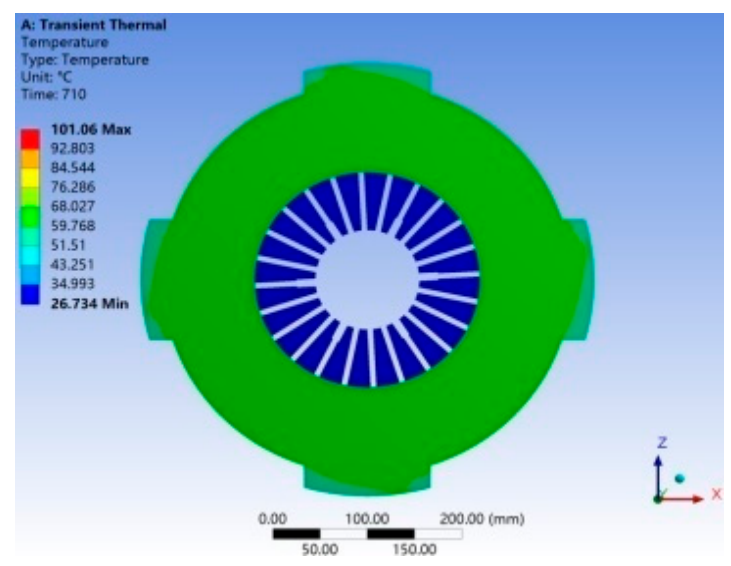

(a)

Figure 6. Cont. 


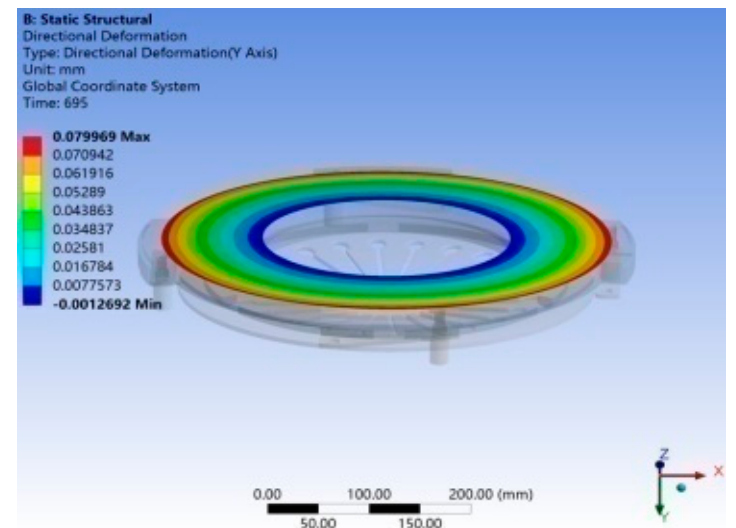

(b)

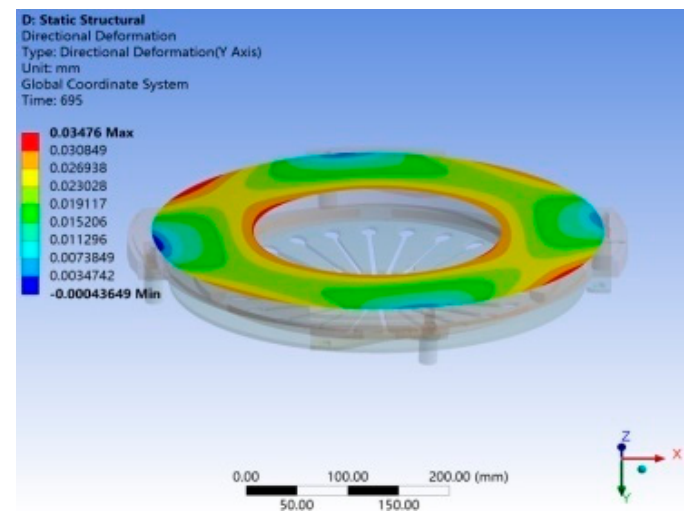

(c)

Figure 6. The temperature field (a), deformation field under constraint condition 1 (b), and deformation field under constraint condition 2 (c) of pressure plate at $695 \mathrm{~s}$.

When the slipping time is $60 \mathrm{~s}$, the temperature field on the friction surface of the pressure plate expands along the radial direction, see Figure 5, as the heat flux of friction surface is proportional to the radius of the surface. Meanwhile, the maximum temperature of the pressure plate is located on the outer rim of the friction surface. From Figure 5, note that the pressure plate presents an internal convex shape deformation under both the first and second types of constraint conditions. This is consistent with the actual deformation of the clutch plate when it is heated during the slip period of engagement. The pressure plate starts the cooling process from 60 s to $3500 \mathrm{~s}$. At $695 \mathrm{~s}$, it can be seen from Figure 6 that the deformation field of the pressure plate presents an inner convex shape under the first type of constraint conditions (shown in Figure 3), whereas the deformation field of the pressure plate presents almost horizontal under the second type of constraint conditions (shown in Figure 4). As the cooling process continues, up to $3500 \mathrm{~s}$, as shown in Figure 7, the bulk temperature of the pressure plate is almost cooled to the room temperature $\left(25^{\circ} \mathrm{C}\right)$ and the friction surface of the pressure plate under the first type of constraint conditions becomes nearly flat (the relative axial displacement on the friction surface is $0.002484 \mathrm{~mm}$ ); however, under the second type of constraint condition, the friction surface of the pressure plate exhibits an obvious concave deformation with the relative axial displacement of $0.186334 \mathrm{~mm}$. 


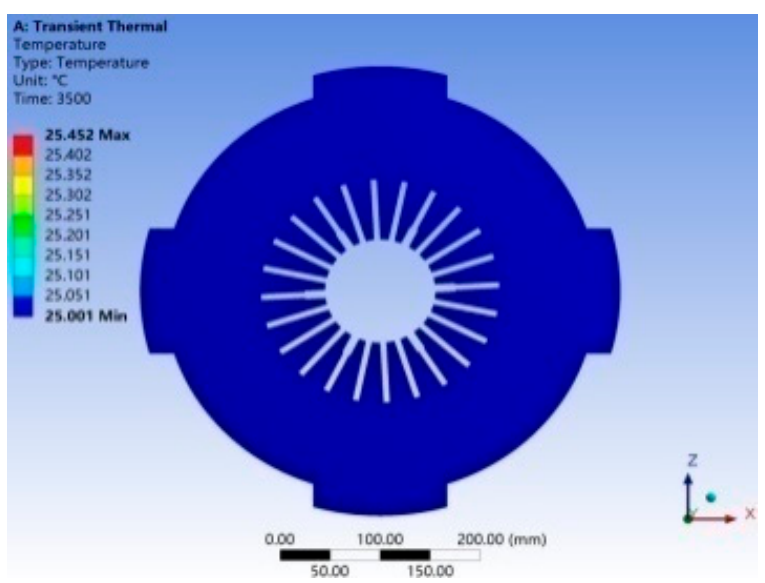

(a)

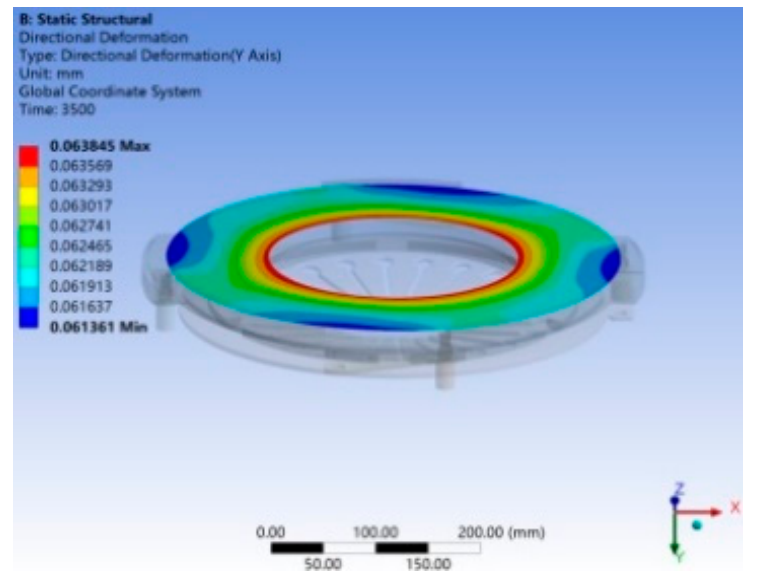

(b)

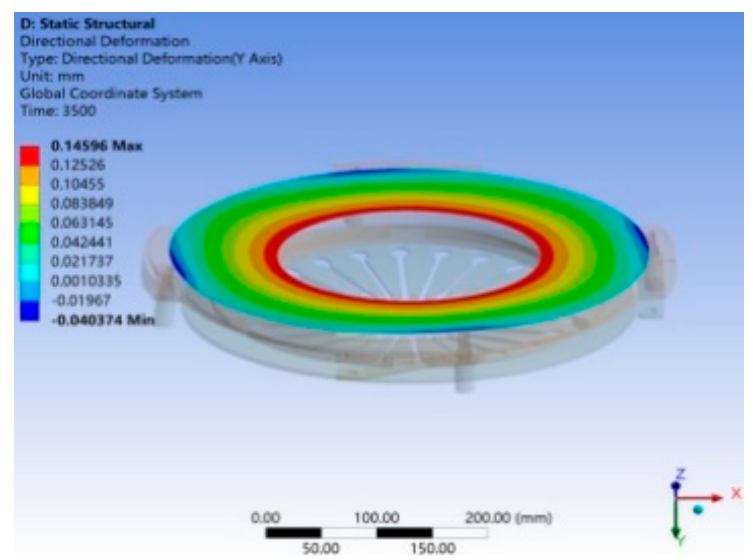

(c)

Figure 7. The temperature field (a), deformation field under constraint condition 1 (b), and deformation field under constraint condition 2 (c) of pressure plate at $3500 \mathrm{~s}$.

The relative axial deformation of the friction surface of the pressure plate over time is shown in Figure 8 and Table 1 under these two different constraint conditions. In Figure 8, the relative axial deformation of the pressure plate under the first type of constraint is highlighted in blue and that under the second type of constraint is in red. 


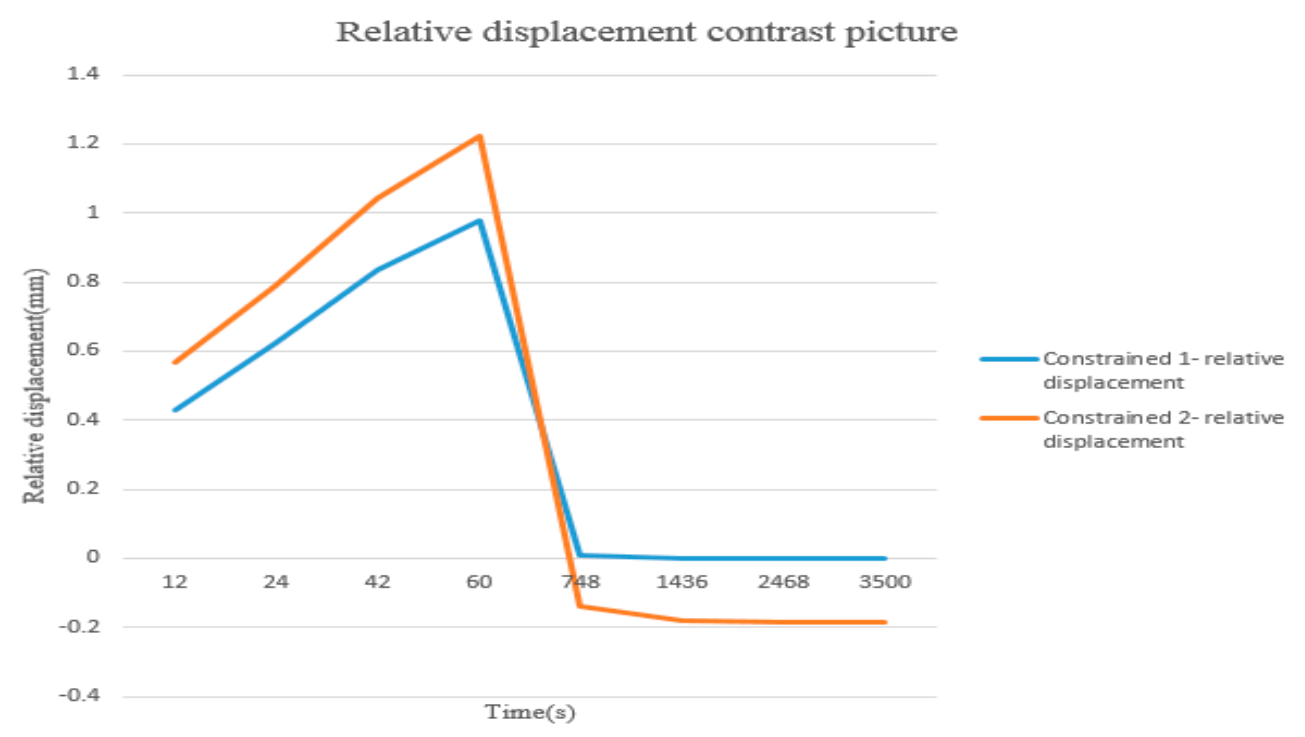

Figure 8. Relative displacement curve of axial deformation at the bottom of pressure plate.

Table 1. Comparison of relative axial displacements under different constraints.

\begin{tabular}{cccc}
\hline Time (s) & Displacement (mm) & $\begin{array}{c}\text { Constraint 1-Relative Axial } \\
\text { Displacement }(\mathbf{m m})\end{array}$ & $\begin{array}{c}\text { Constraint 2-Relative Axial } \\
\text { Displacement }(\mathbf{m m})\end{array}$ \\
\hline 12 & 0.430 & 0.568 \\
\hline 24 & 0.623 & 0.790 \\
\hline 42 & 0.833 & 1.040 \\
\hline 60 & 0.979 & 1.222 \\
\hline 748 & 0.007 & -0.136 \\
\hline 1436 & -0.003 & -0.180 \\
\hline 2468 & -0.003 & -0.186 \\
\hline 3500 & -0.003 & -0.185 \\
\hline
\end{tabular}

From Figure 8 and Table 1, it can be seen that during the slipping period ( $0-60 \mathrm{~s})$, the relative axial displacement (inner convex deformation) of the friction surface of the pressure plate increases with time. At the earlier stage of the cooling process, the inner convex deformation of the pressure plate decreases. The distinguished difference of the relative axial displacement of the pressure plate between the first type of constraint and the second type of constraint takes place at the later stage of the cooling process. At some point, specifically, at time $748 \mathrm{~s}$ for the first type of constraints, the relative axial displacement of the pressure plate is nearly zero, meaning the pressure plate returns to its original flat shape, and from that time on the pressure plate stays horizontal. However, for the second type of constraints, the pressure plate also returns back to its original horizontal state at some moment before $748 \mathrm{~s}$, but continually changes as the cooling process progresses. Ultimately, the pressure plate shows an inner concave deformation with a steady value of $\sim 0.18 \mathrm{~mm}$ at the end of the cooling process. This tendency of axial deformation of the pressure plate here is exactly consistent with that of the rig test in [1] and our empirical data. Note that this is the first time the inner concave deformation of a pressure plate is obtained by the numerical simulation to date.

\subsection{Stress Field Analysis of Pressure Plate}

To better understand the fundamental mechanism of this inner concave deformation of pressure plate, we placed an emphasis on the equivalent von Mises stress distribution of the pressure plate is 
emphasized. Particularly, the equivalent von Mises stresses at four key points of the cross section of the pressure plate, labeled as a, b, c, and d in Figure 9, were analyzed, where, a and b are the upper corner points of the cross section, $\mathrm{c}$ is the inner corner point of the cross section, and $\mathrm{d}$ is the outer corner point of the cross section of the pressure plate.

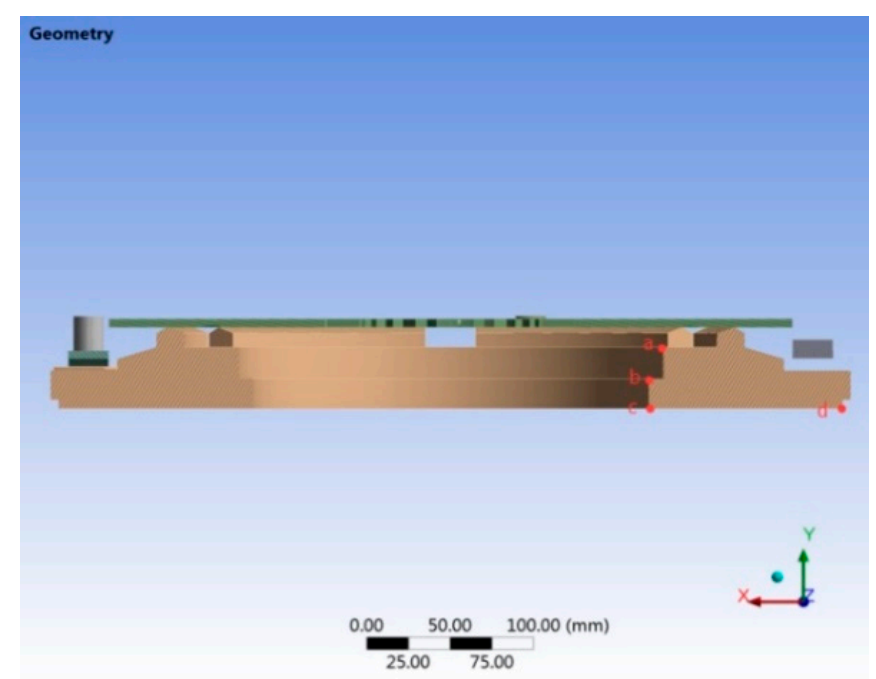

Figure 9. Key points on the pressure plate as well as the axial and chassis surfaces.

Through the finite element analysis, the equivalent von Mises stress of the above four key points as a function of time under the first type of constraint conditions is obtained, as shown in Figure 10 and Table 2. The equivalent von Mises stress under the second type of constraint conditions is also obtained, as shown in Figure 11 and Table 3. The comparison of the stress for the two types of constraints is shown in Figure 12.

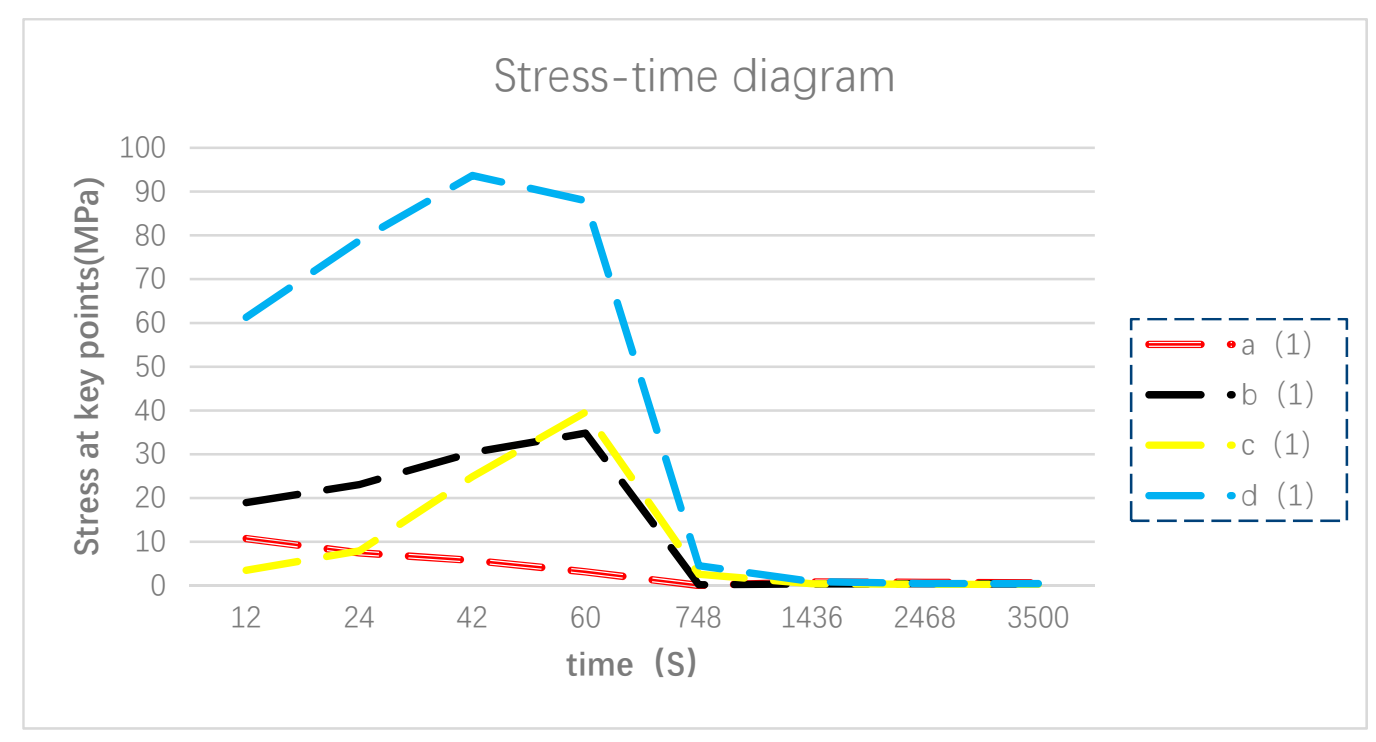

Figure 10. Stress at the four critical points of the pressure plate under the first type of constraint. 
Table 2. Stresses at the four critical points of the pressure plate under the first type of constraint.

\begin{tabular}{cccccc}
\hline \hline \multirow{2}{*}{ Time (s) } & Von Mises Stress at Key Points (MPa) & $\mathbf{a}$ & $\mathbf{b}$ & $\mathbf{c}$ & $\mathbf{d}$ \\
\hline \multirow{2}{*}{12} & 10.754 & 18.950 & 3.527 & 61.312 \\
\hline 24 & 7.500 & 23.084 & 7.944 & 78.827 \\
\hline 42 & 5.772 & 30.400 & 24.875 & 93.675 \\
\hline 60 & 3.164 & 34.827 & 39.643 & 87.925 \\
\hline 748 & 0.045 & 0.198 & 2.587 & 4.530 \\
\hline 1436 & 0.847 & 0.385 & 0.424 & 0.939 \\
\hline 2468 & 0.757 & 0.415 & 0.300 & 0.457 \\
\hline 3500 & 0.672 & 0.408 & 0.301 & 0.454 \\
\hline
\end{tabular}

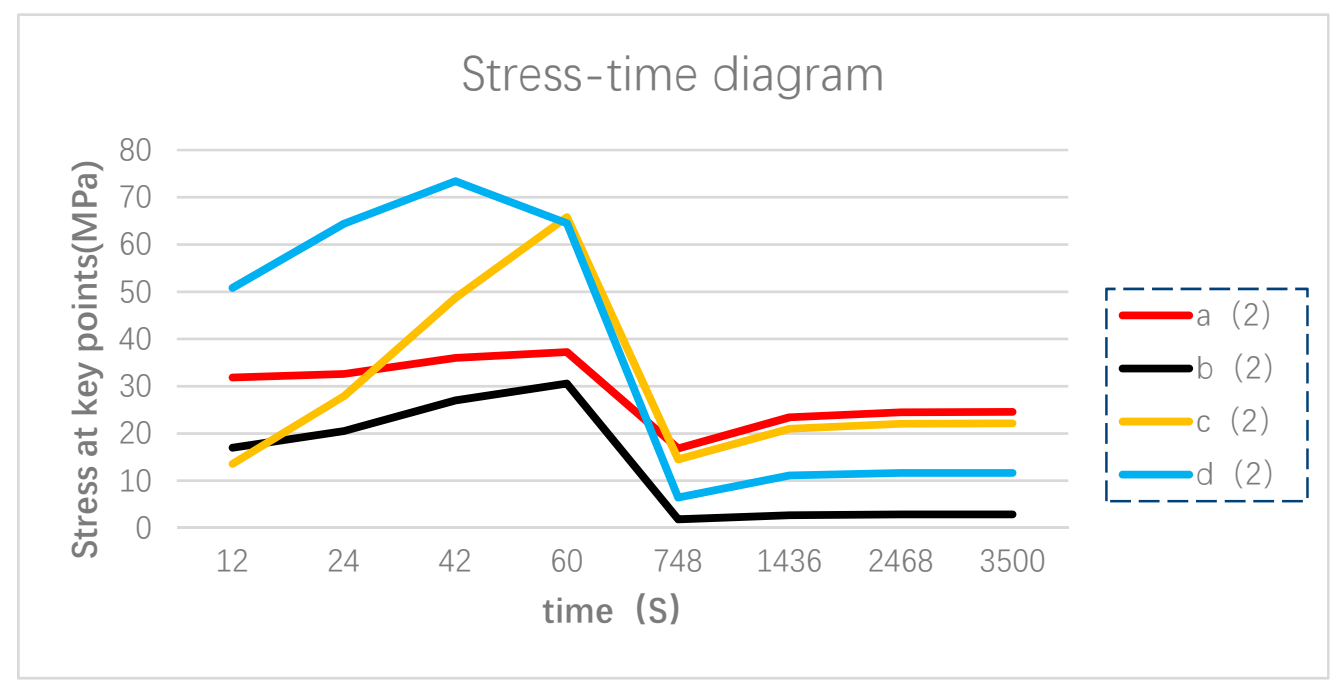

Figure 11. Stress at the four critical points of the pressure plate under the second type of constraint.

Table 3. Stresses at the critical points of the pressure plate under the second type of constraint.

\begin{tabular}{|c|c|c|c|c|c|}
\hline Time (s) & Stress at Key Points (MPa) & a & b & c & d \\
\hline & 12 & 31.834 & 16.982 & 13.566 & 50.807 \\
\hline & 24 & 32.592 & 20.522 & 27.863 & 64.417 \\
\hline & 42 & 35.987 & 26.997 & 48.689 & 73.416 \\
\hline & 60 & 37.227 & 30.551 & 65.793 & 64.478 \\
\hline & 748 & 16.822 & 1.8175 & 14.516 & 6.4262 \\
\hline & 1436 & 23.431 & 2.7072 & 20.983 & 11.112 \\
\hline & 2468 & 24.489 & 2.866 & 22.050 & 11.626 \\
\hline & 3500 & 24.585 & 2.877 & 22.147 & 11.620 \\
\hline
\end{tabular}

It can be seen from Figures 10-12 that the tendency of the equivalent von Mises stress of the four key points of the pressure plate is generally the same as that of the relative axial displacement of the pressure plate shown in Figure 8 for each type of constraints; that is, the equivalent von Mises stress increases during slipping (heating) and decreases during separation (cooling).

For the first type of constraints, at $\sim 748 \mathrm{~s}$, the equivalent von Mises stresses at the all four points decrease to $5 \mathrm{MPa}$ (seen in Table 2), and the difference between these stresses value is very small. Starting from $748 \mathrm{~s}$, the equivalent von Mises stresses of the four key points are further reduced to $\sim 1 \mathrm{MPa}$, and they are almost uniform at $\sim 0.5 \mathrm{MPa}$ (seen in Table 2) later. This quantitative trend of 
the equivalent von Mises stresses is also identical to that of the relative axial displacement of friction surface of the pressure plate, indicating that the displacement of the pressure plate is determined by its stress state.

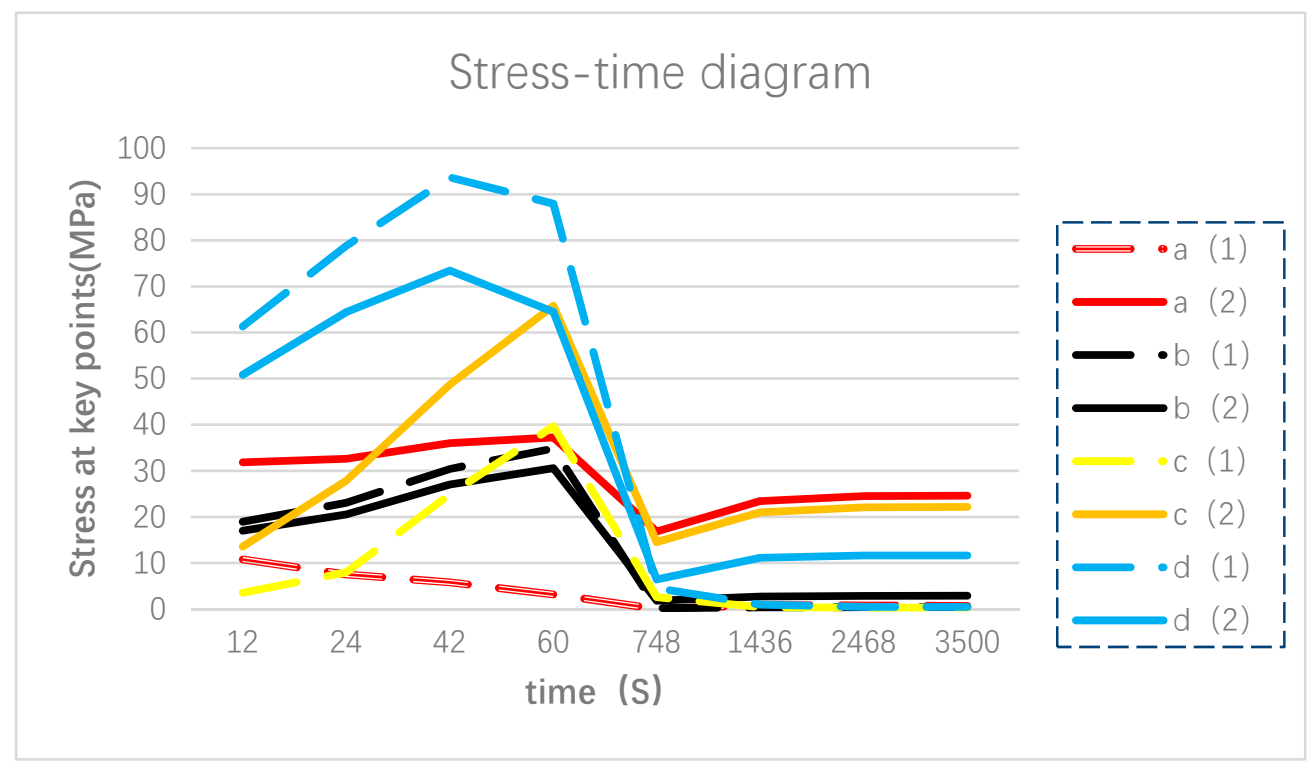

Figure 12. Comparison of the stress for the two types of constraints: 1: the first constraints; 2 the second constraints.

For the second type of constraints, the stress state of these four key points shows a significant difference during the natural cooling process compared with the first type of constraints. Unlike the first type of constraints, at $\sim 748 \mathrm{~s}$, the equivalent von Mises stresses at the four points are relatively high and the difference between these four stress values is also obvious (see Table 3). Particularly, at $748 \mathrm{~s}$ and later, theses four equivalent von Mises stresses are also relatively high and not uniform, as under the first type of constraints. This indicates that these differences in the equivalent von Mises stress cause the differences in the displacement for the pressure plate. It is confirmed that the concave shape displacement is induced by the overstress state.

The equivalent von Mises stress distributions in the entire cross section of the pressure plate at $3500 \mathrm{~s}$ under the first constraints and the second constraints are shown in Figures 13 and 14, respectively. In Figures 13 and 14, the geometry in gray is the undeformed geometry and the one in color is the deformed geometry under the prescribed load. The pressure plate has almost returned to its original horizontal location under the first constraints, and, meanwhile, the equivalent von Mises stress in the cross section of the pressure plate is very low and almost uniform (shown in Figure 13). For the second constraints, the pressure plate shows a concave shape deformation and the equivalent von Mises stress in the cross section is relatively high with a changed location (shown in Figure 14).

More specifically, under the second constraints, the von Mises stress of point a (represents the upper part of the inner rim of the pressure plate) is higher than that of the other three points (represents the lower part of the inner rim and part of outer rim, respectively) when the concave shape of the pressure plate occurs. This specific stress state causes the concave shape of the deformed pressure plate.

The stress state of the cross section of the pressure plate is directly related to its boundary conditions. When the pressure plate cools, the temperature of the pressure plate decreases and the pressure plate tends to "shrink". Under the second boundary constraints, where the radial degrees of freedom $(\mathrm{DoF})$ of the outer edge of the pressure plate is constrained and the inner rim of the pressure plate is relatively free, the inner rim of the pressure plate will shrink along the radial and axial directions. As the stress in the upper part of the inner rim is higher than that in the lower part of the inner rim of the pressure plate, the inner rim shrinks away from the lower part of inner rim in the axial 
direction. Consequently, this shrinkage results in the concave shape deformation of the pressure plate. On the contrary, under the first boundary constraints, the inner rim and the outer rim of the pressure plate can shrink freely along the radial direction during the cooling process and therefore the concave shape deformation does not occur.

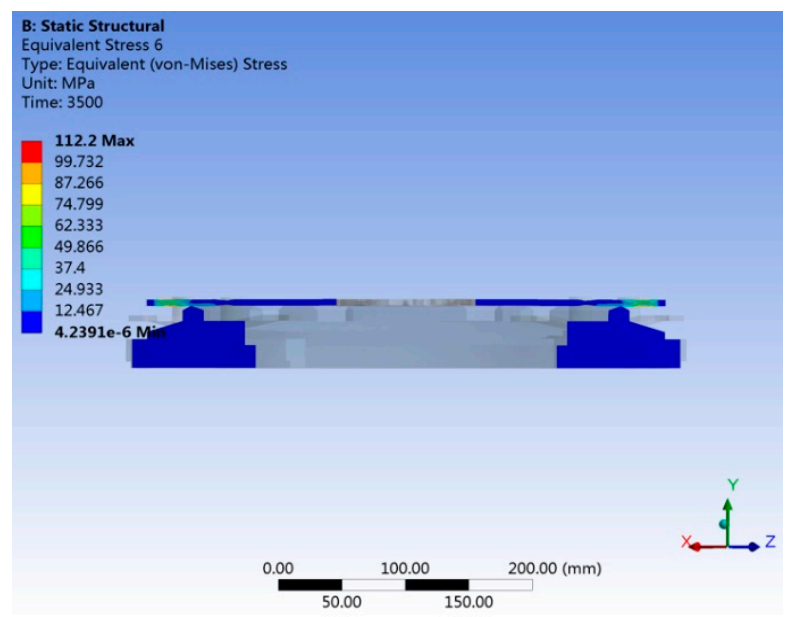

Figure 13. Stress distribution of pressure plate under constraint 1.

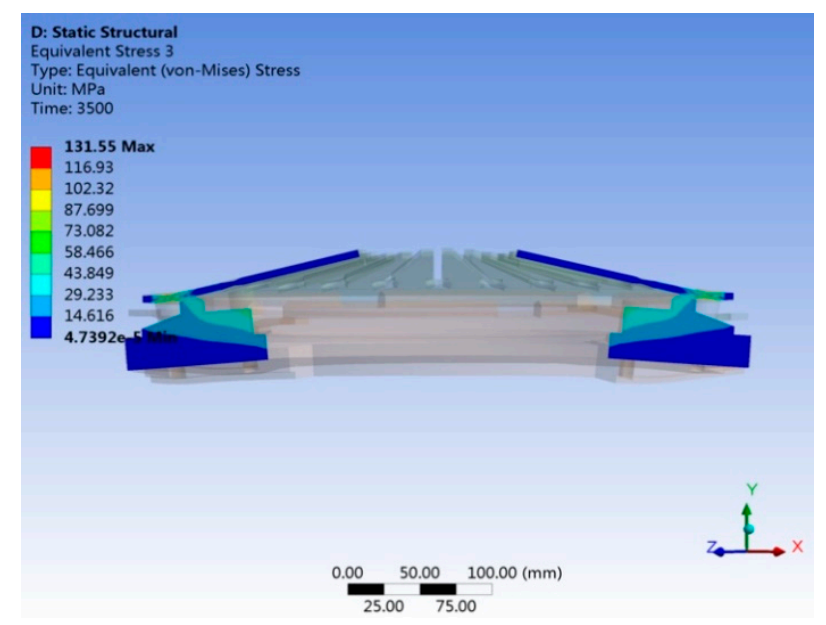

Figure 14. Stress distribution of pressure plate under constraint 2.

\section{Conclusions}

A finite element model of the assembly of automotive $\varphi 430$ pressure plate, including the pressure plate, diaphragm springs, rivets, as well as transmission plates, is developed. The thermal displacement distribution of the pressure plate under continuous slipping followed by a natural cooling process is simulated based on the assembly model. The concave shape deformation of the pressure plate during the cooling process is obtained for the first time by the numerical simulation. It is found that this particular concave shape deformation of pressure plate is determined by its thermal stress distribution. When the equivalent von Mises stress of the upper part of the inner rim of the pressure plate is higher than that of the lower part of the inner rim and that of the outer rim of the pressure plate, concave shape deformation occurs. The equivalent von Mises stress of pressure plate is strongly affected by the boundary conditions of pressure plate. In addition, the critical stress state can easily be achieved when the outer rim of the pressure plate in radial direction is constrained.

Author Contributions: Conceptualization, Y.G. and Y.-B.Y.; methodology, P.W.; software, W.G., writing-Original draft preparation, W.G.; writing-Review and editing, Y.-B.Y.; visualization, P.W.; supervision, Y.G. 
Funding: This research was funded by National Natural Science Foundation of China (No. 51965012) and the Key Research and Development Plan of Guangxi Province of China (No. AB18126002).

Conflicts of Interest: The authors declare no conflict of interest.

\section{References}

1. Lu, F.; Zhu, M.T.; Liu, X.L. Transient thermal analysis and the structure improvement of a clutch pressure plate. J. Transp. Sci. Eng. 2013, 2, 94-97. (In Chinese)

2. Xu, X.K. Research on Clutch Failure of Minicar. Ph.D. Thesis, Wuhan University of Technology, Wuhan, China, 2008. (In Chinese).

3. Yantian, S.S.; Zhu, T.N. Solving the thermal deformation problem of clutch pressure plate Introduction to testing technology of Daikin Research Institute. Foreign Car 1986, 4, 33-35. (In Chinese)

4. Zhang, T.S.; Zhu, M.T. Finite element analysis of thermal deformation and thermal stress of automobile clutch pressure plate. Automob. Technol. 1995, 5, 23-26. (In Chinese)

5. Zhang, W.B.; Lai, L.F.; Yan, S.R. Finite element design of clutch pressure plate based on ANSYS. Chin. J. Eng. Mach. 2007, 4, 426-429. (In Chinese)

6. Zhu, M.T.; Liu, X.L.; Gu, Y.X. Coupling heat structure analysis of the pressure plate of clutch in vehicle start procession. In Proceedings of the Annual Meeting of China Society of Automotive Engineering 2013, Beijing, China, 26 November 2013. (In Chinese).

7. Abdullah, O.I.; Schlattmann, J. Finite element analysis of temperature field in automotive dry friction clutch. Tribol. Ind. 2012, 34, 206-216.

8. Abdullah, O.I. Effect of band contact on the temperature distribution for dry friction clutch. Tribol. Ind. 2013, 35, 317-329.

9. Muhammad, M.J.A.; Abdullah, O.I.; Josef, S. Transient thermoelastic analysis of dry clutch system. Mach. Des. 2013, 5, 141-150.

10. Abdullah, O.I. Stresses and deformations analysis of a dry friction clutch system. Tribol. Ind. 2013, 35, 155-162.

11. Zhang, F.; Bao, J.P. Finite element analysis and improvement of thermal stress of the truck clutch pressure plate. For. Mach. Wood Work. Equip. 2011, 4, 23-26. (In Chinese)

12. Fu, J.R.; Wang, Q.C.; Hou, Y. Analysis of Temperature distribution on Heavy-Duty Truck's Clutch Pressure-Plate. Comput. Simul. 2012, 10, 336-340. (In Chinese)

13. Gong, Y.B.; Jin, C.; Pan, S.Y. Research on the thermal deformation of the clutch pressure plate and its improvement under the extreme conditions. Mach. Des. Manuf. 2017, 3, 82-84. (In Chinese)

14. Gong, Y.B.; Ge, W.C.; Yi, Y.B. Finite element analysis of thermal buckling characteristics of automotive 430 dry clutch pressure plate. Int. J. Veh. Des. 2018, 78, 108-130. [CrossRef]

15. Xing, Y.E.; Gao, Y.D.; Zhang, G.B. Temperature rise analysis of friction plate of shift clutch. Mod. Manuf. Technol. Equip. 2007, 5, 17-19. (In Chinese)

16. Jin, C.; Gong, Y.B.; Pan, S.Y.; Zhang, D.F. Influence of the Thermal Parameters and the Structural Parameters on the Performance of Clutch Pressure Plate. In Proceedings of the 2015 2nd International Forum on Electrical Engineering and Automation (IFEEA 2015), Guangzhou, China, 26 December 2015.

17. Chen, W.H.; Xu, G.L.; Jin, S.P. Heat Transfer; Wuhan University of Technology Press: Wuhan, China, 2004. (In Chinese)

(C) 2019 by the authors. Licensee MDPI, Basel, Switzerland. This article is an open access article distributed under the terms and conditions of the Creative Commons Attribution (CC BY) license (http://creativecommons.org/licenses/by/4.0/). 